\title{
Correspondence
}

\section{Candida albicans lung abscess sensitive to fluconazole and responding only to amphotericin B in a non-immunocom- promised critically ill patient}

To the Editor:

We would like to present an uncommon case of candida lung abscess in a non-immunocompromised patient who developed disseminated candidiasis and candida albicans lung abscess responding only to amphotericin B despite sensitivity to fluconazole. A 53-yr-old male was transferred to our intensive care unit (ICU) from the floor after developing high fever, respiratory failure requiring mechanical ventilation, bilateral basal infiltrates on his chest $x$-ray and blood cultures growing Candida Albicans sensitive to fluconazole. The patient had undergone a meningioma excision 53 days previously. Postoperative course was complicated by ventilator-associated pneumonia and herpetic meningoencephalitis which were treated with cefuroxime, ceftazidime, vancomycin and acyclovir. No steroids were administered and the patient received total parenteral nutrition for 11 days. On the floor and subsequently in the ICU the patient was placed on iv high-dose fluconazole (800 mg daily) and on empiric broad spectrum antibiotic coverage due to known colonization with methicillin-resistant staphylococcus aureus and acinetobacter baumanii. In the following nine days the blood cultures continued growing C. albicans and the patient remained febrile despite therapy with fluconazole. No candida was grown from intravascular catheters. A thoraco-abdominal computerized tomography revealed multiple cavitary lung lesions consistent with lung abscesses (Figure). Fundoscopy revealed findings consistent with C. albicans endophthalmitis and C. albicans was isolated from the bronchoalveolar lavage fluid. Both staining for acid-fast bacteria and cultures were negative for M. tuberculosis. The patient was switched to amphotericin B on the ninth day of his ICU stay (60 mg daily) and became afebrile two days later. Blood cultures became negative the next day after amphotericin B was administered and remained negative until the patient was discharged in good condition with significant improvement of the lung legions. This course contrasts recent studies in which high-dose fluconazole appeared equivalent to standard dose

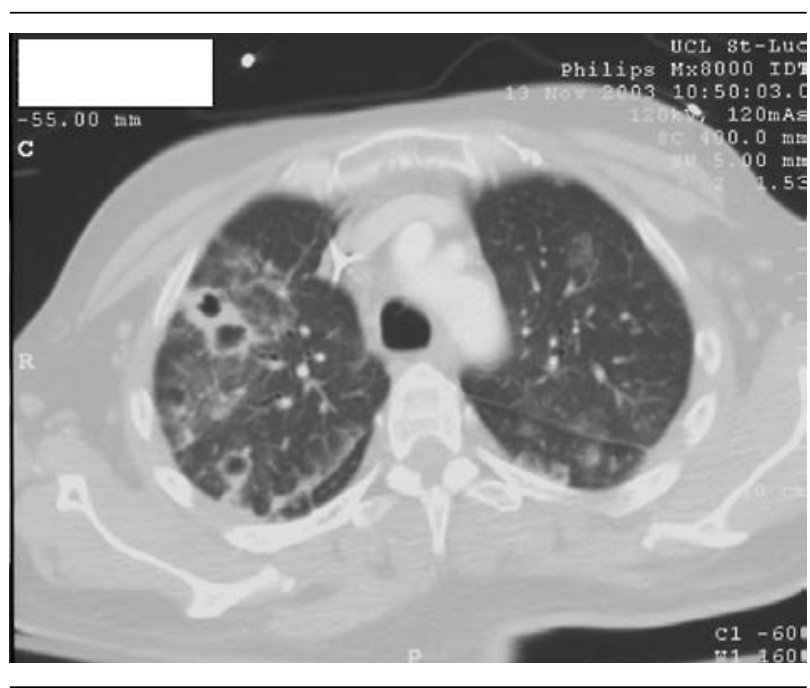

FIGURE Axial contrast-enhanced chest computed tomography image obtained at the level of lung apices shows at lung window settings (WW: $1600 \mathrm{HU}$, WL: $-600 \mathrm{HU}$ ) cavitary lesions located at the upper part of the right upper lobe. Non-specific ground glass opacities are present on both lungs.

amphotericin B. ${ }^{1}$ The persistence of our patient's lesions during therapy with broad-spectrum antibiotic therapy and their resolution after appropriate antifungal therapy points towards the diagnosis of candida lung abscess, a very rare clinical entity. ${ }^{2,3}$ The negative cultures of the intravascular catheters argue against septic candida lung emboli. Additionally, poor tissue penetration of a candida lung abscess and the predominantly fungistatic activity of fluconazole against C. albicans in vitro may explain the poor response to fluconazole. ${ }^{4}$ Clinicians taking care of critically ill patients with documented C. albicans infection sensitive to fluconazole should consider switching to amphotericin B if high-dose fluconazole therapy is not effective and especially if there is a suspicion of a developing lung abscess.

\section{Antonios Liolios MD}

Benjamin Gressens MD

Ingrid Leonard $\mathrm{MD}$

Emmanuel Coche MD

Philippe Hantson MD PhD

Université catholique de Louvain, Brussels, Belgium

E-mail: hantson@rean.ucl.ac.be 


\section{References}

1 Torres HA, Kontoyiannis DP, Rolston KV. High-dose fluconazole therapy for cancer patients with solid tumors and candidemia: an observational, noncomparative retrospective study. Support Care Cancer 2004; 12: $511-6$.

2 Sibvo EI, Vilkko PS, Salminen JT, et al. Subacute primary Candida lung abscess. Scand J Infect Dis 1999; 31: 592-5.

3 Mori T, Ebe T, Takahashi M, et al. Lung abscess: analysis of 66 cases from 1979 to 1991. Intern Med 1993; 32: $278-84$.

4 van Etten EW, van de Rhee NE, van Kampen KM, et al. Effects of amphotericin B and fluconazole on the extracellular and intracellular growth of Candida albicans. Antimicrob Agents Chemother 1991; 35: 2275-81.

\section{Reduction of blood product transfu- sions during liver transplantation}

To the Editor:

We wish to suggest a technique for reducing red blood cell (RBC) transfusions during liver transplantation. Previously, correction of coagulation defects with plasma transfusions did not decrease the need for intraoperative RBC transfusion during liver transplantations. ${ }^{1}$ On the contrary it had produced a hypervolemic state that resulted in an increase of shed blood. As well, plasma and RBC transfusions have been associated with a decreased one-year survival rate..$^{2-4}$ The aim of the present prospective survey was to evaluate whether anesthesiologists could reduce intraoperative RBC transfusions during liver transplantation by changing their anesthesia practice, more specifically by maintaining a low central venous pressure (CVP), by restricting volume replacement, by eliminating all plasma transfusions and by using intraoperative phlebotomy during the transplantation.

With the approval of our hospital Ethics Committee, 61 liver transplantations were prospectively studied during a one-year period and were compared to a retrospective series (1998-2002). ${ }^{1}$ A low CVP was maintained in all patients prior to the anhepatic phase. Coagulation disorders were not corrected. Phlebotomy and cell savers (CS) were used following pre-established criteria $\left(\mathrm{Hb} \geq 85 \mathrm{~g} \cdot \mathrm{L}^{-1}\right.$, normal renal function, hemodynamic stability and enough potential blood loss to prime the CS). The purpose of the phlebotomy at the beginning of the case was to reduce the CVP. Hemodilution was not used, which also avoided coagulation factor dilutions.

The mean number of intraoperative $\mathrm{RBC}$ units transfused per patient was $0.3 \pm 0.7$. No plasma, nor platelets, nor cryoprecipitate were transfused. Seventynine percent of the patients received no blood products during their liver transplantation. The CVP was lowered significantly from an average of $12.0 \pm 3.9$

TABLE Comparison of two groups: 206 patients (1998-2002) and 61 patients (2002-2003)

\begin{tabular}{llll}
\hline Variables $^{*}$ & 206 patients (1998-2002) & 61 patients (2002-2003) & $P$ Value \\
\hline Sex $(\mathrm{male})$ & $62.6 \%$ & $60.7 \%$ & $\mathrm{NS}$ \\
Age $(\mathrm{yr})$ & $52 \pm 11$ & $51 \pm 14$ & $\mathrm{NS}$ \\
Weight $(\mathrm{kg})$ & $73 \pm 17$ & $69 \pm 15$ & $\mathrm{NS}$ \\
Height $(\mathrm{cm})$ & $166 \pm 9$ & $167 \pm 10$ & $\mathrm{NS}$ \\
Starting Hb value $\left(\mathrm{g} \cdot \mathrm{L}^{-1}\right)$ & $105.3 \pm 22.4$ & $105.5 \pm 23.6$ & $\mathrm{NS}$ \\
Starting INR value & $1.8 \pm 0.9$ & $1.6 \pm 0.5$ & $\mathrm{NS}$ \\
Starting platelet count $\left(109 \mathrm{pl} \cdot \mathrm{L}^{-1}\right)$ & $102 \pm 68$ & $100 \pm 47$ & $\mathrm{NS}$ \\
Starting creatinine value $\left(\mathrm{mol} \cdot \mathrm{L}^{-1}\right)$ & $107 \pm 72$ & $97 \pm 59$ & $\mathrm{NS}$ \\
Pugh's score & $9.9 \pm 2.3$ & $9.4 \pm 2.3$ & $\mathrm{NS}$ \\
MELD score & $17 \pm 10$ & $17 \pm 9$ & $\mathrm{NS}$ \\
RBC transfused (un/pt) & $2.8 \pm 3.5$ & $0.3 \pm 0.7$ & $<0.0001$ \\
Final Hb value $\left(\mathrm{g} \cdot \mathrm{L}^{-1}\right)$ & $87.3 \pm 13.9$ & $84.6 \pm 18.5$ & $\mathrm{NS}$ \\
Blood loss $(\mathrm{mL})$ & $1479 \pm 1750$ & $912 \pm 468$ & 0.0254 \\
Crystalloids before clamping $(\mathrm{mL})$ & $1531 \pm 426$ & $1050 \pm 314$ & $<0.0001$ \\
Creatinine 24 hr postoperatively $\left(\mu \mathrm{mol} \cdot \mathrm{L}^{-1}\right)$ & $107 \pm 50$ & $97 \pm 62$ & $\mathrm{NS}$ \\
Length of hospitalisation $($ days) & $47 \pm 34$ & $45 \pm 32$ & $\mathrm{NS}$ \\
\% of phlebotomy & $0 \%$ & $39 \%$ & $<0.0001$ \\
Survival rate at one year $(\%)$ & $91.4 \%$ & $<0.0001$ \\
\hline
\end{tabular}

*Values are mean $\pm S D$, otherwise percentage $(\%)$, statistical analysis was performed by using Student $\mathrm{t}$ test or Welch $\mathrm{t}$ test or Chi-square $\mathrm{t}$ test as appropriate. $\mathrm{Hb}=$ hemoglobin; INR = international normalization ratio; $\mathrm{MELD}=$ model of end liver-stage disease; $\mathrm{RBC}=$ red blood cells. 